Acta Crystallographica Section A

Foundations of Crystallography

ISSN 0108-7673

Received 28 September 2007 Accepted 16 November 2007

(C) 2008 International Union of Crystallography Printed in Singapore - all rights reserved

\section{Electron crystallography: imaging and single-crystal diffraction from powders}

\author{
Xiaodong $\mathrm{Zou}^{\mathrm{a}, \mathrm{b} *}$ and Sven Hovmöller \\ ${ }^{a}$ Structural Chemistry, Stockholm University, SE-106 91 Stockholm, Sweden, and ${ }^{\mathbf{b}}$ Berzelii \\ Centre EXSELENT on Porous Materials, Stockholm University, SE-106 91 Stockholm, Sweden. \\ Correspondence e-mail: zou@struc.su.se
}

\begin{abstract}
The study of crystals at atomic level by electrons - electron crystallography - is an important complement to X-ray crystallography. There are two main advantages of structure determinations by electron crystallography compared to X-ray diffraction: (i) crystals millions of times smaller than those needed for X-ray diffraction can be studied and (ii) the phases of the crystallographic structure factors, which are lost in X-ray diffraction, are present in transmissionelectron-microscopy (TEM) images. In this paper, some recent developments of electron crystallography and its applications, mainly on inorganic crystals, are shown. Crystal structures can be solved to atomic resolution in two dimensions as well as in three dimensions from both TEM images and electron diffraction. Different techniques developed for electron crystallography, including threedimensional reconstruction, the electron precession technique and ultrafast electron crystallography, are reviewed. Examples of electron-crystallography applications are given. There is in principle no limitation to the complexity of the structures that can be solved by electron crystallography.
\end{abstract}

\section{Introduction}

Electron diffraction (ED) of crystals was discovered in 1927, only 15 years after the discovery of X-ray diffraction. Soon after, the fact that electrons, unlike X-rays, can be focused into an image was exploited in the invention of the electron microscope (Knoll \& Ruska, 1932). The ability to form images in the electron microscope makes it very powerful for looking at non-crystalline as well as crystalline objects. Indeed, electron microscopy (EM) could reveal details within living cells, with a resolution 100 times higher than the light microscope (Porter et al., 1945) and played a crucial role in the birth of cell biology in the 1950s. For crystals, the situation was different. On one hand, inorganic crystals mostly have unit cells smaller than $10 \AA$ and so could not be studied until the resolution of the EM surpassed the $4 \AA$ point-to-point resolution in around 1970. Protein crystals, on the other hand, have much larger unit cells but are often too thick ( $>100 \mathrm{~nm}$ ) to be studied by EM and they are very radiation sensitive.

The first transmission-electron-microscopy (TEM) images of an inorganic crystal, the metal oxide $\mathrm{Ti}_{2} \mathrm{Nb}_{10} \mathrm{O}_{29}$ at $3 \AA$ resolution (Iijima, 1971), were almost simultaneous with the first protein crystals studied by TEM: bacteriorhodopsin (7 $\AA$ ) and catalase (9 ̊) (Unwin \& Henderson, 1975). Yet the two fields of studies of crystals by electrons, inorganics and proteins, for many years evolved with regrettably little contact. The protein field followed the pioneering work at the MRC Laboratory of Molecular Biology in Cambridge (DeRosier \& Klug, 1968; Henderson \& Unwin, 1975; Unwin \&
Henderson, 1975) using Fourier-transform-based image processing to get both the crystallographic structure-factor amplitudes and phases from the TEM images and retrieve the structures of membrane proteins. For inorganic crystals, such crystallographic image processing was recognized much later, although the image resolution for inorganic crystals is much higher, near atomic resolution. There was an unfortunate misunderstanding caused by unclear definitions of the word 'phase', which is used for both the 'phases' of crystallographic structure factor and 'phases' of electron waves. The phase information of the crystallographic structure factors is present in TEM images, while the phases of electron waves forming the TEM images are lost. It suffices to mention the different periodicities related to the two totally different entities: the periodicity for each crystallographic structure factor is the $d$ value, ranging from sub-ångström up to the unit-cell dimensions. The periodicity of the electron wave is the wavelength of the electrons, often less than $0.03 \AA$. The mix-up of these two meanings of the word phase has caused great confusion in the inorganic field of electron microscopy. The phase confusion problem has been investigated and clarified in detail by Zou (1999). The phases of crystallographic structure factors can be obtained by crystallographic image processing (Klug, 1978-79; Hovmöller et al., 1984; Zou et al., 1996) from one or several TEM images. The phase of the electron wave at the exit surface of a crystal can be obtained by through-focus exitwave reconstruction, from a series of TEM images taken at different defocus values (Van Dyck \& Op de Beeck, 1990; Coene et al., 1992, 1996). 
Similar to X-ray diffraction, the phase information is also lost in electron diffraction. Already around 1950, a group of scientists in Moscow made great contributions in using electron diffraction data for structure determination of both organic and inorganic crystals (Pinsker, 1949; Vainshtein, 1956; Zvyagin, 1967). The Patterson method and model building were used for structure solution. Later, Dorset \& Hauptman (1976) showed that direct methods could also be used for phasing ED data. Cowley $(1953 a, b, c)$ performed structure analysis by electron diffraction before he developed the theory of multibeam dynamical scattering together with Moodie (Cowley \& Moodie, 1957, 1959a,b). The theory showed that electron diffraction intensities deviate greatly from those produced by kinematical scattering. This almost led to a consensus that it was impossible to obtain intensities in electron diffraction patterns that were kinematical or nearkinematical, i.e. could be treated as closely related to the crystallographic structure-factor amplitudes, except possibly for crystals containing only light elements, such as organic or biological crystals. Thus, little effort was made, except by the Moscow group (Pinsker, 1949; Vainshtein, 1956; Zvyagin, 1967; Vainshtein et al., 1992), Cowley (1953a,b,c) and Dorset (1995), to collect high-quality ED data and to quantify ED data at all. This is in great contrast to X-ray crystallography, where the importance of relative intensities of diffraction spots was recognized immediately by Bragg and Bragg in 1912. We now know that it is possible to solve crystal structures from electron diffraction data with rather heavily distorted intensities. As long as the strongest reflections remain the strongest, the Patterson function and direct methods may well give a correct crystal structure. This has been demonstrated by Dorset $(1995,1998)$ and shown to hold also for quite thick inorganic crystals by Weirich et al. (2000).

TEM instrumentation has continued to develop also during the past decade, especially in terms of resolution. Field emission guns (FEGs) provide much more coherent and bright electrons and have pushed the information limit in the TEMs to sub-ångström levels.

Over the last 25 years, it has been our ambition to develop electron crystallography into a mature technique such that unknown complicated crystal structures can indeed be solved by electron crystallography, from ED data or high-resolution transmission electron diffraction (HRTEM) images alone, or by combining amplitude data from ED with phases from HRTEM images. The invention of the electron precession technique by Vincent \& Midgley (1994) made it possible to collect more diffraction spots from a single electron diffraction pattern and obtain integrated intensities from a single crystal, similar to the electron texture patterns from polycrystalline samples by the Moscow group (Zvyagin, 1967). This new technique has finally brought the two communities within TEM studies of inorganic compounds - crystallographers and physicists - together. Many are now enthusiastic about the possibilities of collecting high-quality near-kinematical electron diffraction data.

In this paper, we show some recent examples of electroncrystallography applications, mainly on inorganic crystals, with some of the many exciting developments in the field during the last decade or so. For electron crystallography studies of organic crystals and proteins, we refer to recent reviews by Dorset $(1998,2007)$ and Renault et al. (2006). Surface structure analysis by electron crystallography was reviewed by Leslie et al. (1999). Examples of the study of charge density by convergent-beam electron diffraction (CBED) are given by Zuo et al. (1999) and $\mathrm{Wu}$ et al. (2004), where multibeam dynamical scattering has been taken into account. Electron holography is another technique for studying crystals, which gives access to the structure information encoded in the phase of the electron wave and is especially useful for materials with electric and magnetic properties. For this technique, we refer to books and reviews by Tonomura (1999), Völkl et al. (1999) and Lichte et al. (2007). There are many other techniques within electron microscopy that are useful in the study of crystals, including electron-energy-loss spectroscopy and energy-dispersive X-ray analysis, but they are outside the scope of this paper.

\section{Structure determination of inorganic crystals from HRTEM images}

\subsection{Direct interpretation of HRTEM images}

Atoms can be observed directly by TEM. However, HRTEM images are sensitive to focus, astigmatism, and crystal thickness and orientation. Only images of very thin samples that can be considered as nearly weak phase objects ( $<5 \mathrm{~nm}$ for metals and alloys, slightly more for oxides and other compounds mainly containing light elements), taken near the optimal, so-called Scherzer, defocus can be directly interpreted in terms of structure projections. Furthermore, crystals have to be well aligned. The point-to-point resolution of a conventional TEM operated at $200-400 \mathrm{kV}$ is about $1.6-$ $2.0 \AA$; enough to resolve metals in oxides but not the $\mathrm{O}$ atoms. Recent development of aberration-corrected TEM by Haider et al. (1998) has meant dramatic improvements and pushed images to sub-ångström resolution. Such HRTEM images allow quantitative atomic scale analysis of crystals, crystal interfaces and defects, where $\mathrm{O}$ atoms and $\mathrm{O}$ vacancies can be visualized directly by TEM (Jia et al., 2003; Jia \& Urban, 2004).

Scanning transmission electron microscopy (STEM) can provide dark-field images using an annular detector. Highangle annular dark-field (HAADF) images give a contrast that directly relates to the atomic numbers of the atomic columns: $Z$-contrast (Pennycook \& Jesson, 1991). Aberration correction in STEM has also pushed the resolution of $Z$-contrast imaging to sub-ångström resolution (Nellist et al., 2004).

The new sub-ångström techniques in TEM promise very exciting results in the future. However, for crystal-structure determinations, the results are still mainly from conventional TEM and electron diffraction. The power of TEM compared to diffraction techniques is most evident in samples that are not perfectly crystalline. Crystals showing diffuse scattering can only be described in general terms from diffraction data 
but a HRTEM image shows every atom column. One such case is illustrated in Fig. 1; a barium niobium oxide with a complex pattern of niobium octahedra on an underlying simple tetragonal crystal lattice (Svensson, 1990; Köhler et al., 1992; Zubkov et al., 1994). The position of every metal column can be seen in the HRTEM image. When the Fourier transform of the micrograph is calculated, a diffractogram is seen. All the regions show diffuse scattering with largely similar features but it is of course impossible to deduce the structure from these diffraction patterns.

\subsection{Retrieval of projected potential by image processing}

In general, HRTEM images are distorted by electron optics (defocus, astigmatism, beam misalignment), crystal misalignment and dynamical scattering. Often, a HRTEM image is not exactly an enlarged image of the projected structure and cannot be interpreted directly in terms of the structure projection. The effects of the electron optics can be described using a contrast transfer function (CTF). The CTF depends both on the electron-beam coherency and on the defocus value and astigmatism; the former results in a damping of the image information at high resolution. The FEG electron sources provide a brighter electron beam and more monochromatic and coherent electrons, resulting in much more high-resolution information in HRTEM images. HRTEM images from a FEG TEM can contain information on crystal structures even beyond $1 \AA$. However, the contrast within a HRTEM image depends on many parameters including the defocus and astigmatism, as well as the crystal thickness and crystal misalignment. Even for HRTEM images taken at the Scherzer resolution from a very thin crystal (weak phase object), the contrast at the resolution beyond the Scherzer resolution can only be interpreted after applying image processing, compensating for the CTF effects.

Image processing can be done on single images, according to the crystallographic image processing method originally suggested by Klug (DeRosier \& Klug, 1968; Klug, 1978-79) and further developed by Unwin \& Henderson (1975) and Hovmöller et al. (1984; Hovmöller, 1992) or by combining data from several HRTEM images taken at different defocus values using the through-focus exit-wave reconstruction method developed by Coene et al. $(1992,1996)$ to retrieve the complete exit wavefunction of electrons at the exit surface of the crystal. The crystallographic image-processing method mainly aims at retrieving the structure-factor amplitudes and phases of the crystal in order to obtain the crystal-structure projection. It can be applied only to very thin crystals. On the other hand, the structure-factor amplitudes and phases obtained from HRTEM images of different crystals and orientations, by crystallographic image processing, can easily be combined to get a better structure projection (Zou et al., 1996) or three-dimensional potential map, and can also be combined with X-ray diffraction data, as described in $\$ 2.4$. The
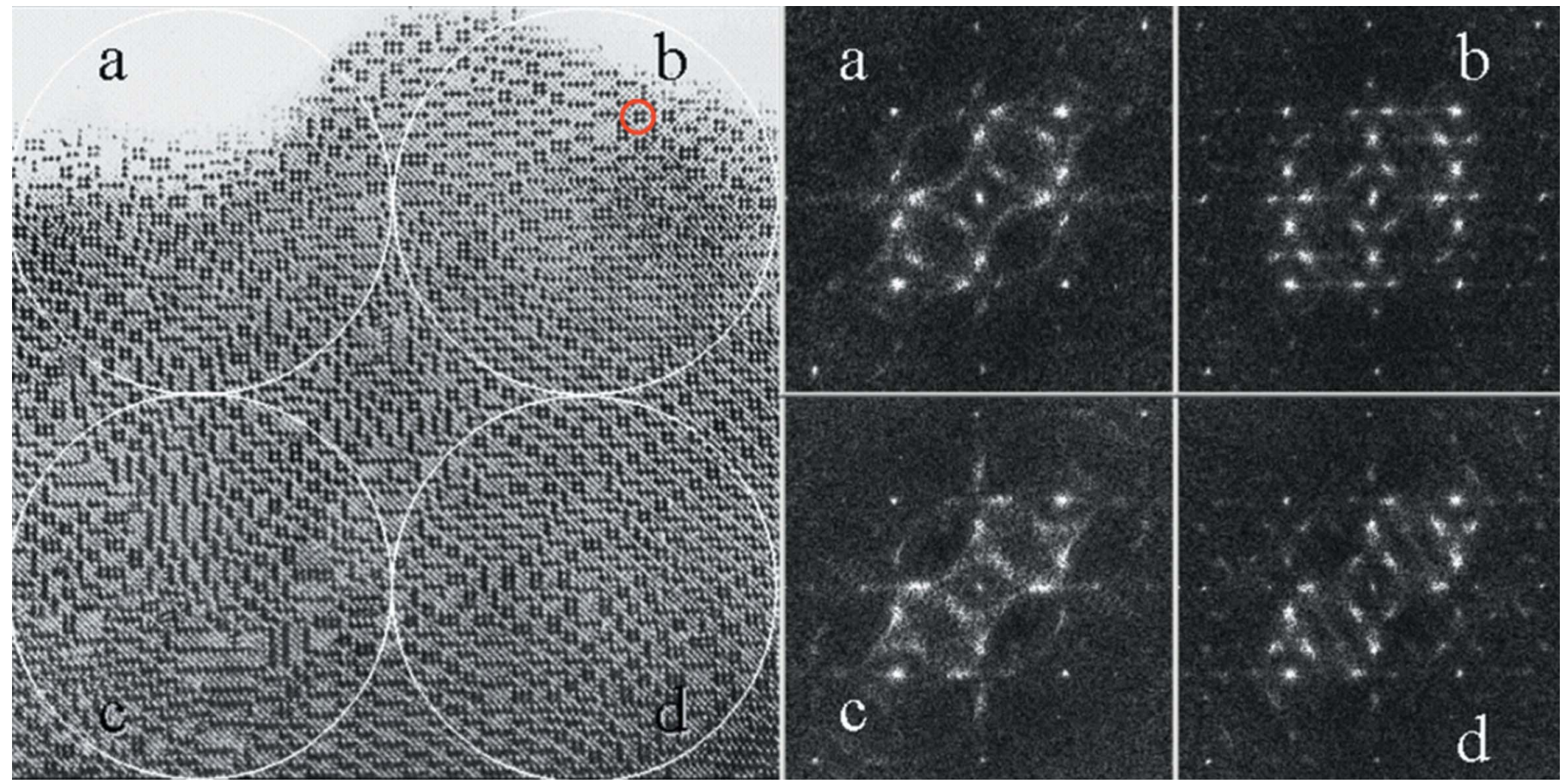

Figure 1

HRTEM image of a barium niobium oxide with diffractograms calculated from four different regions $(a)-(d)$. In the image, every single column of metal atoms is seen as a black dot. The larger black crosses are octahedra of six $\mathrm{Nb}$ atoms. Four such octahedra, in a $2 \times 2$ arrangement, are marked with a red circle in $(b)$. The sample is an example of a non-stoichiometric phase in the interesting region between crystalline and amorphous; only the underlying tetragonal structure is crystalline. The HRTEM image is by courtesy of Gunnar Svensson, Stockholm University, with permission. 


\section{feature articles}

through-focus exit-wave reconstruction method aims at retrieving the electron wavefunction at the exit surface of the sample, which contains information from both the crystal structure and interaction between the crystal and electron wave. This method can be applied for thicker crystals and is especially useful for studying defects and interfaces (Zandbergen et al., 1997) at sub-ångström resolution. It is also possible to reach sub-ångström resolution by electron holography (Lichte et al., 2007). The images reconstructed from the through-focus exit-wave reconstruction and electron holograph, if applicable, can be further processed by crystallographic image processing to improve the images for crystalstructure determination. It is worth pointing out that using the image-processing methods to retrieve the sub-ångström resolution is more economically favorable than using an aberra- tion-corrected TEM but requires good knowledge of the methods.

The crystallographic image-processing method was first used for solving crystal structures of a number of niobium oxides (Hovmöller et al., 1984; Wang et al., 1988; Zou et al., 1996). In the projected potential maps obtained by crystallographic image processing from experimental HRTEM images at $2.5 \AA$ resolution, all metal atoms were resolved and the atomic positions determined with an accuracy of $0.1-0.2 \AA$. Li Fan-hua and Fan Hai-fu's group in Beijing developed an image-processing method, combined with image deconvolution and phase extension by maximum entropy and direct methods, to solve the crystal structure of $\mathrm{K}_{2} \mathrm{Nb}_{14} \mathrm{O}_{36}$ (Hu et al., 1992). They also applied direct methods for solving unconventional crystal structures such as quasicrystals (Xiang et al.,
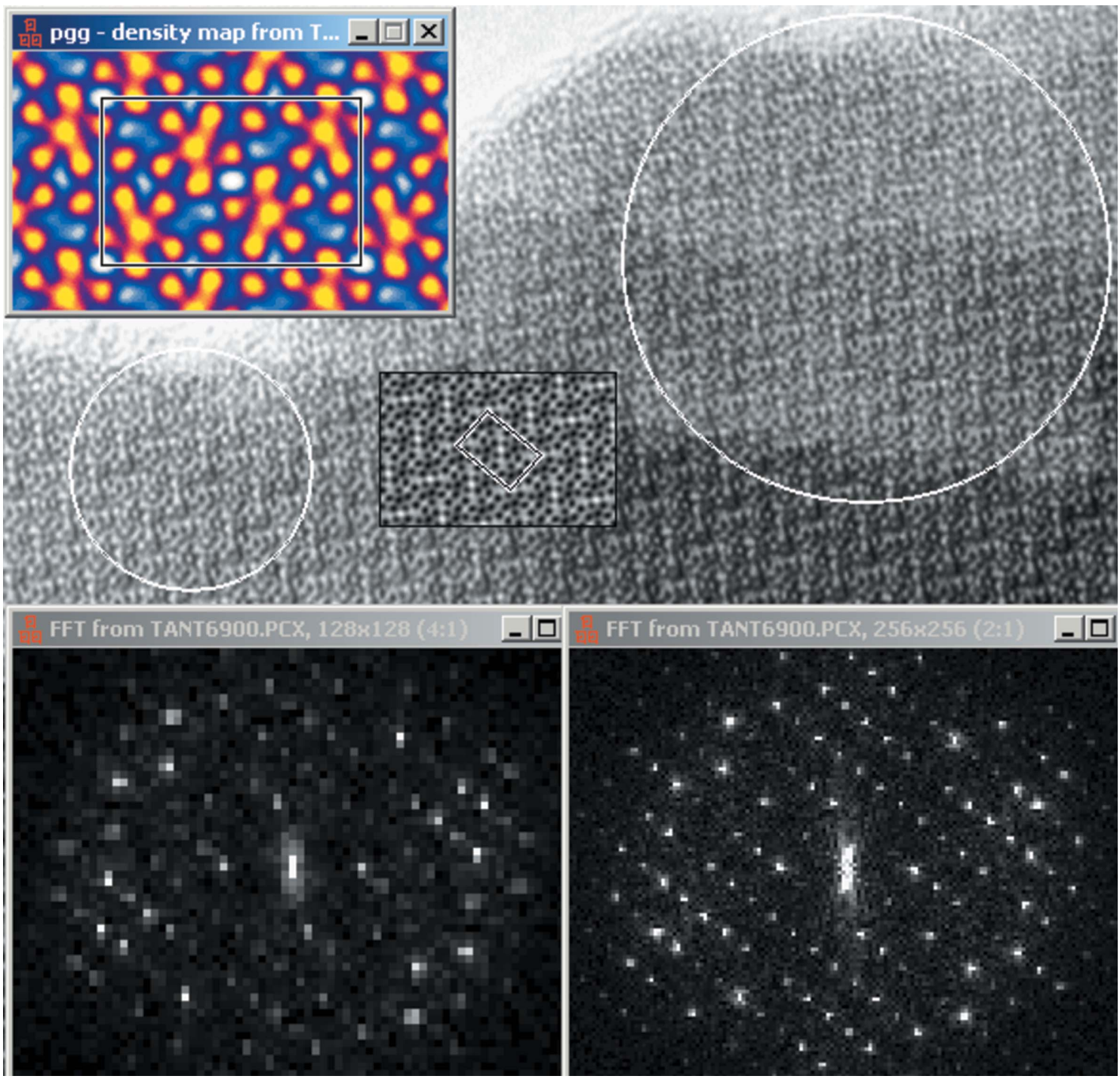

\section{Figure 2}

An electron-microscopy image of a crystal of $\mathrm{Li}_{2} \mathrm{NaTa}_{7} \mathrm{O}_{19}$ taken with a standard $200 \mathrm{kV}$ TEM. Two thin circular regions have been cut out and their Fourier transforms calculated (below). The larger region is $168 \AA$ across, the smaller $84 \AA$ and contains only 15 unit cells. As expected, the diffractogram of the larger area (right) shows sharper peaks, but it is possible to solve the structure even from the smaller region (left), as seen in the insets with one unit cell marked. The $\mathrm{Nb}$ atoms are shown in black in the smaller inset and orange in the bigger inset. Thus, a structure can be solved from a crystal smaller than $10 \times 10 \mathrm{~nm}$ using electron crystallography. 
1990) and incommensurately modulated structures (Mo et al., 1992).

It is possible to solve crystal structures from extremely small crystals using HRTEM. The heavier metal-atom positions in an oxide can be found from crystals as small as ten unit cells. An example is shown in Fig. 2, where most of the Ta atoms in $\mathrm{Li}_{2} \mathrm{NaTa}_{7} \mathrm{O}_{19}$ form characteristic filled pentagonal clusters.

Crystal tilt is a common problem in HRTEM images due to crystals being slightly misaligned or bent. The amplitudes of reflections far away from the tilt axis are attenuated by the tilt (Zou, 1995). However, the phases are practically unaffected for small tilts and thin crystals (Zou et al., 1995; Hovmöller \& Zou, 1999). Crystal tilt can be compensated to some extent by imposing the crystallographic symmetry into the image.

\subsection{Three-dimensional structure by combining HRTEM images from different projections}

HRTEM images are only projections of the three-dimensional structure. Most structure determinations of inorganic compounds by HRTEM have been on crystals with at least one short unit-cell axis ( $<5 \AA$ ). Such structures can be solved from a single projection along that short axis, where at least the heavy atoms do not overlap. Unfortunately, in most cases the physical dimensions of a crystal are inversely proportional

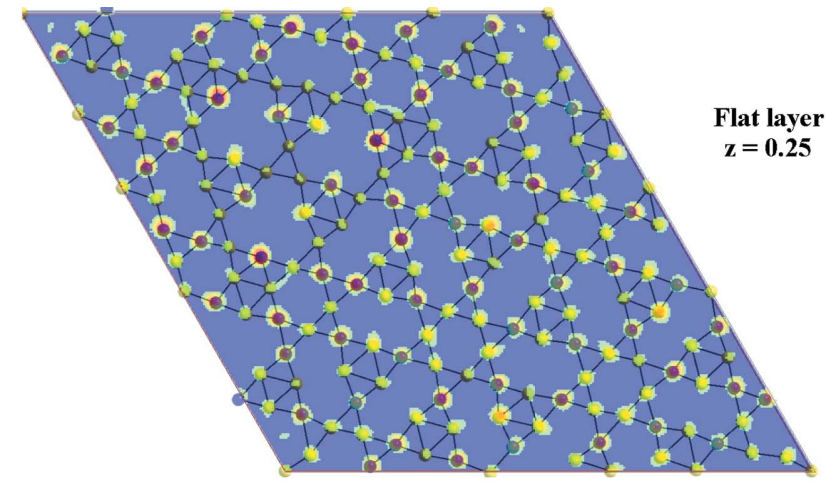

(a)

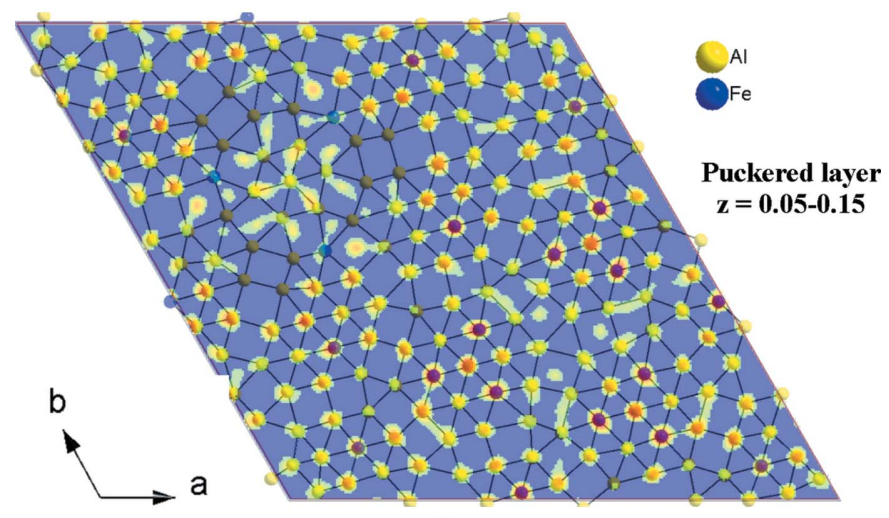

(b)

Figure 3

Two sections of the three-dimensional potential map of $v$-AlCrFe obtained by electron crystallography with the structure superimposed. All atoms are clearly resolved and it is possible to distinguish transition metals ( $\mathrm{Fe}, \mathrm{Cr}$, strong peaks) from $\mathrm{Al}$ (weak peaks). From Zou et al. (2003). to the unit-cell parameters, so that a crystal with one short unit-cell parameter (for example $a=4, b=20, c=25 \AA$ ) typically grows as needles with the longest physical dimension parallel to the short unit-cell dimension. Thus, it is often hard to prepare crystal fragments that are thin enough in the most desired directions. For complex structures with large unit-cell axes, atoms overlap (exactly or nearly) in any projection direction and may not be resolved even with an aberrationcorrected TEM of a resolution beyond $1 \AA$. The only way to solve the overlapping problem is to collect several images from different directions and combine these images into a three-dimensional structure. In three dimensions, atoms are never closer than the interatomic distances, typically $1-2 \AA$. Nowadays, standard TEMs provide images with resolutions around $1.6 \AA$. This resolution is sufficient to resolve almost all atoms in inorganic samples. Thus, it is possible to solve crystal structures at atomic resolution by TEM, provided sufficiently many projections are collected for a three-dimensional data set. This can often be achieved from several different crystals with different orientations on the TEM grids, although in practice it is cumbersome to collect reasonably complete three-dimensional data.

The first three-dimensional structures determined by TEM at around $25 \AA$ resolution were biological complexes, such as the helical tail of bacteriophage T4 (DeRosier \& Klug, 1968) and spherical viruses (Crowther et al., 1970). At this resolution, individual protein molecules, but not their internal structures, could be discerned. A remarkable breakthrough was the first near-atomic structure of a membrane protein; the three-dimensional structure of bacteriorhodopsin which was solved by electron microscopy to $7 \AA$ resolution, revealing the seven trans-membrane $\alpha$-helices (Henderson \& Unwin, 1975). This was nearly ten years before the first membrane protein was solved by X-ray crystallography. In Berkeley, Wenk et al. (1992) combined for the first time HRTEM images from different projections of a mineral, the silicate staurolite, and constructed a three-dimensional electron potential map. In this map, all atoms ( $\mathrm{Fe}, \mathrm{Al}, \mathrm{Si}$ and $\mathrm{O})$ were clearly resolved. This work showed that three-dimensional electron crystallography has a great potential also in structure determination of inorganic crystals - perhaps even more promising for inorganic structures than for organic and biological structures because of the lower radiation sensitivity and thus higher resolution.

Electron crystallography has made important contributions to the study of ordered mesoporous materials. In these materials, the pores are ordered in a crystalline manner but the walls are constructed of amorphous silica, carbon or metal oxides. The ordering range is from a few to a few tens of nanometres and very few diffraction peaks can be detected by $\mathrm{X}$-ray powder diffraction. Three-dimensional reconstruction of HRTEM images from different projections has been applied to solve the three-dimensional structures of a series of ordered mesoporous materials, for example MCM-41 (Ia $\overline{3} d)$, SBA-6 $(P m \overline{3} n)$, SAB-16 $(\operatorname{Im} \overline{3} m)$ and AMS- $n$, mostly by Terasaki and co-workers (Sakamoto et al., 2000, 2002, 2004; Kaneda et al., 2002; Garcia-Bennett, Terasaki et al., 2004; 
Garcia-Bennett, Miyasaki et al., 2004; Gao et al., 2006). It has also been used for studying the pore structure changes of ordered large cage-type mesoporous silica FDU-12s under different synthesis temperatures (Yu et al., 2006).

The structure of the very complex intermetallic compound $v$-AlCrFe with $a=40.687, c=12.546 \AA$, space group $P 6_{3} / m$, was solved by the combination of HRTEM images and selected-area electron diffraction patterns from 13 zone axes (Zou et al., 2003). 124 of the 129 unique atoms (1176 in the unit cell) were found in the remarkably clean three-dimensional potential maps obtained by electron crystallography (Fig. 3). Until today, this is the most complicated structure solved to atomic resolution by electron crystallography. It demonstrates that there is no limit to determine complex structures by electron crystallography.

\subsection{HRTEM combined with X-ray powder diffraction}

Electron crystallography is especially important for structure determination of crystalline powders, i.e. crystallites in the (sub)micrometre range. These are too small for singlecrystal X-ray diffraction, even on a synchrotron, and so are typically studied by X-ray powder diffraction. However, it is very hard to solve unknown crystal structures by X-ray powder diffraction if the unit-cell dimensions are large, say all are larger than $10 \AA$ and/or the sample is not pure - especially if it contains three or more compounds. Zeolites are examples of such structures. Electron crystallography has been used for structure determination of unknown zeolite structures (see, for example, Leonowicz et al., 1994; Liu et al., 2001). During the last year, two zeolite structures TNU-9 and IM-5 that could not be solved by X-ray powder diffraction alone were solved by combining HRTEM images from three different projections and X-ray powder diffraction data (Gramm et al., 2006; Baerlocher et al., 2007). Crystallographic structurefactor phases were obtained from HRTEM images taken along different zone axes using the program CRISP (Hovmöller, 1992). For zeolite TNU-12, the phase information was incorporated directly into the zeolite-specific algorithm FOCUS, which incorporates some of the crystal chemical information used in model building into the structure determination process (Grosse-Kunstleve et al., 1997, 1999). The structure of TNU-9 with 24 unique $\mathrm{Si} / \mathrm{Al}$ and 52 unique $\mathrm{O}$ atoms could thus be solved (Gramm et al., 2006). For the structure determination of the zeolite catalyst IM-5, a more general procedure that can be used for any types of structures was used. The phase information from IM-5 was incorporated into the charge flipping structure-solution algorithm, using the program Superflip (Palatinus \& Chapuis, 2006) to generate the starting phase sets. The structure of IM-5 with 24 unique $\mathrm{Si}$ and 47 unique $\mathrm{O}$ atoms (864 atoms in the unit cell) could be solved (Fig. 4) (Baerlocher et al., 2007). These two structures

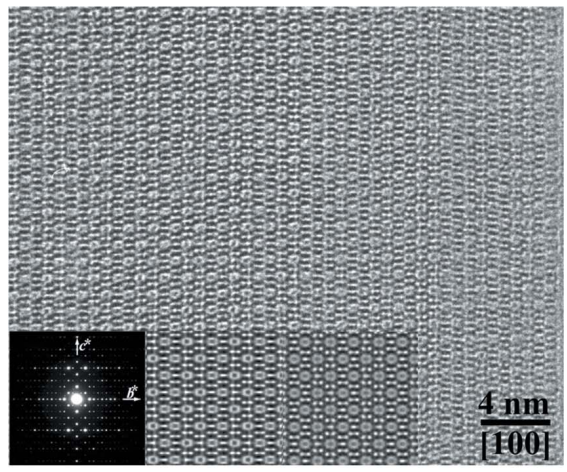

(a)

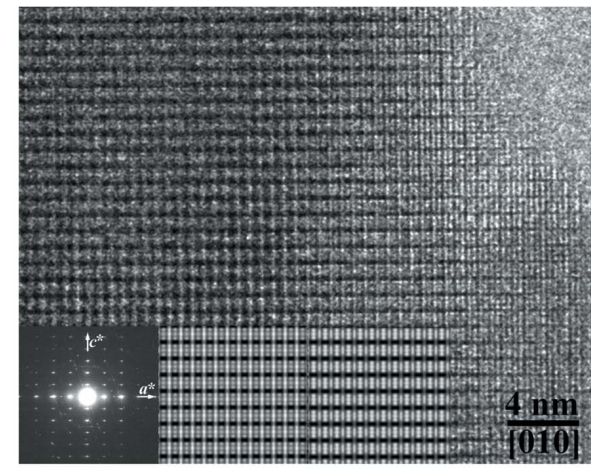

(b)

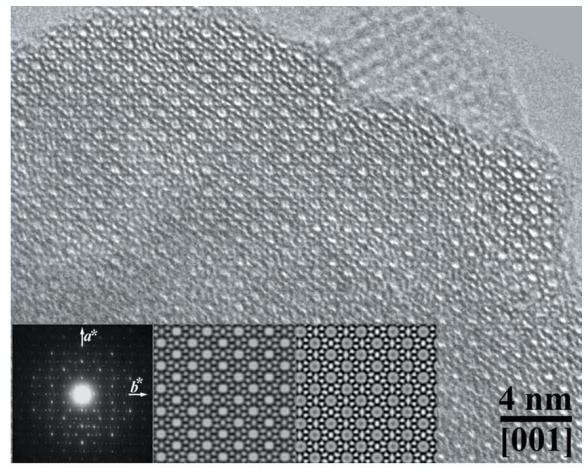

(c)

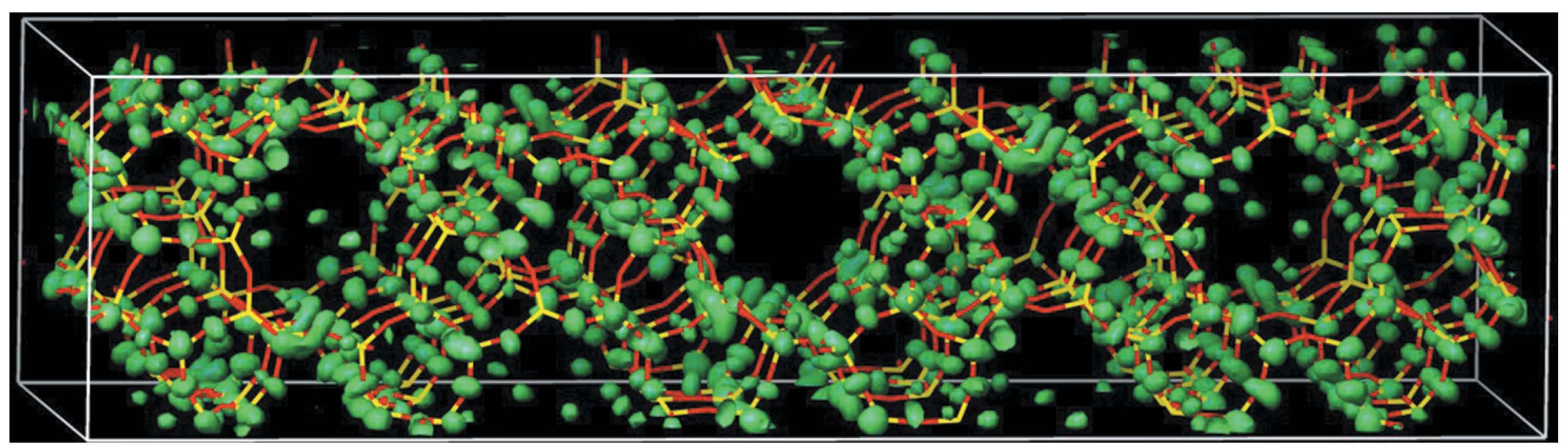

(d)

Figure 4

High-resolution transmission-electron-microscopy images taken along different zone axes of IM-5. (a) [100], (b) [001] and (c) [010]. Shown as insets are the corresponding ED pattern (left), the CTF-corrected and symmetry-averaged image (middle), and the computer simulation from the structural model (right). (d) The final three-dimensional density map (in green) with a stick model of the final structure of IM-5 (Si atoms yellow, O atoms red) superimposed for comparison. From Baerlocher et al. (2007). 
are more complex than any other zeolites solved today, even by single-crystal X-ray diffraction. Recently, we could also solve the structure of zeolite IM-5 only from HRTEM images taken along three different projections, by three-dimensional reconstruction (Sun et al., 2007).

\section{Structure determination from electron diffraction}

\subsection{Data-collection techniques and data reduction}

Owing to multiple scattering, electron diffraction data usually deviate substantially from kinematic data. Sample bending and radiation damage may also affect the data. Thus, data quality and data-collection strategies are most important issues for structure determination from electron diffraction data.

For TEM images, automatic tomography methods have been developed over the last decade and used, for example, in three-dimensional reconstruction of whole cells and nanoparticles. It is possible to automatically take a whole series of around 100 images at for example $1^{\circ}$ intervals. The tilting of the specimen, recentering of the sample and focusing are all done automatically. The three-dimensional structure can be seen in three-dimensional visualization programs. However, the resolution of electron tomography is somewhat limited, to nanometre resolution.

Kolb et al. (2007) have developed a method for automatic electron diffraction tomography, combining scanning transmission electron-microscopy (STEM) imaging with diffraction-pattern acquisition in nanodiffraction mode. This allows automated recording of single-crystal diffraction tilt series from nanoparticles with sizes down to $5 \mathrm{~nm}$. Owing to the low dose used in STEM, organic crystals can also be studied.

The net result of multiple scattering of electrons is that the stronger diffraction spots redistribute their intensity to the weaker spots. Above a critical thickness, all diffraction spots are essentially equally intense (except for the fall-off with scattering angle) and then there is no longer any structural information in the diffraction pattern except for the lattice. One reason for this dynamical behavior is the very short wavelength of electrons; some 50 times shorter than X-rays. This results in an Ewald sphere that is almost flat; in a single still photo along a zone axis, all diffraction spots out to beyond $1 \AA$ are simultaneously excited and thus interact. One way to minimize multiple diffraction is therefore to tilt the crystal by a degree or two. This can be done by electron precession (Vincent \& Midgley, 1994), where the beam is tilted by a few degrees and then rotated in a conical fashion, such that the same area of the sample is always illuminated by the electron beam. Beneath the sample, in a synchronized fashion, the beam is tilted in the opposite direction, such that the diffraction pattern remains stationary. The method is analogous to precession in X-ray crystallography, except that in the X-ray case it is the crystal and film that move while the beam is fixed.

During precession, the Ewald sphere scans through the reflections, which results in integrated intensities. The great improvement in data quality by electron precession can be appreciated from diffraction patterns of the cubic mineral uvarovite, shown in Fig. 5. Not only are the intensities close to kinematical, i.e. close to the squares of the structure-factor amplitudes, they also go to higher resolution when using precession.

\subsection{Unit-cell and space-group determination and indexing from powders}

Unlike X-ray powder diffraction, where the three-dimensional reciprocal lattice collapses into a one-dimensional line spectrum, each electron diffraction pattern gives an undistorted representation of a two-dimensional section through the three-dimensional reciprocal lattice. The unit-cell parameters can be directly determined from one or several ED patterns (Zou et al., 2004) and all reflections can be uniquely indexed. If the crystal diffracts well, a conventional still ED

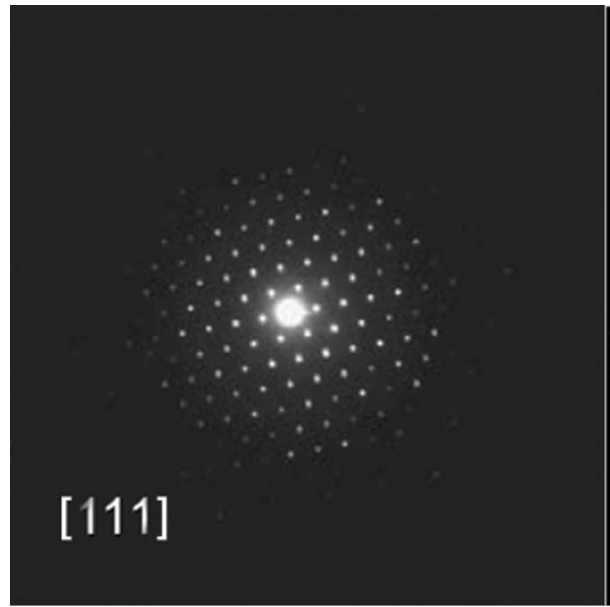

(a)

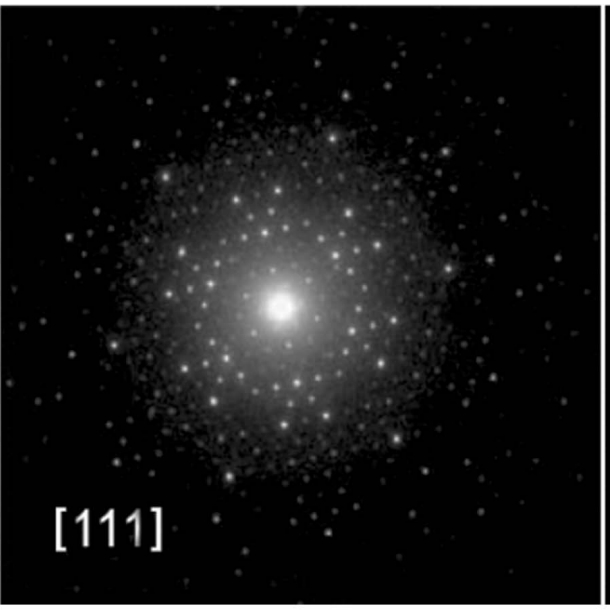

(b)

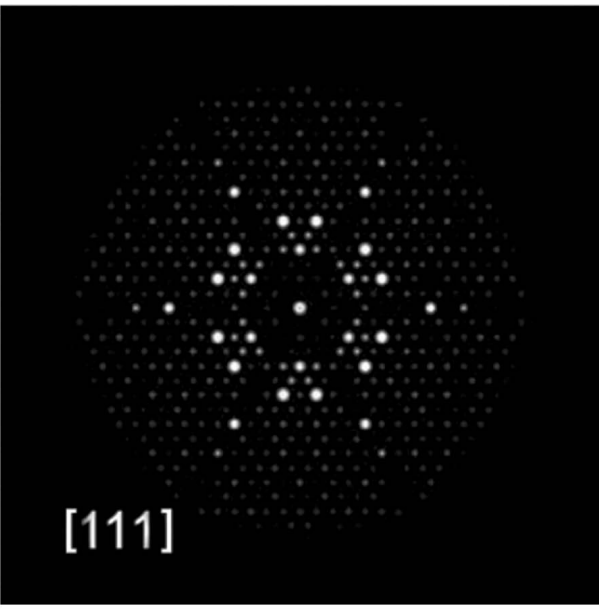

(c)

\section{Figure 5}

Electron diffraction patterns of uvarovite, $\mathrm{Ca}_{3} \mathrm{Cr}_{2}\left(\mathrm{SiO}_{4}\right)_{3}$, space group $I a \overline{3} d, a=11.99 \AA$, with precession $(a)$ off and $(b)$ on. $(c)$ Simulated diffraction pattern, using the kinematical approximation. Notice the effects of dynamical diffraction in $(a)$; all reflections have nearly the same intensities. In contrast, the precession pattern $(b)$ is very close to the kinematically calculated one in (c). After Gemmi \& Nicolopoulos (2007). 
pattern may contain narrow rings of reflections from the highorder Laue zones (HOLZs) around the zero-order Laue zone (ZOLZ). The HOLZs carry additional three-dimensional information from which the unit-cell parameters and lattice types can be determined.

Determination of unit-cell parameters by electron diffraction helps for correct indexing of X-ray powder diffraction data. On the other hand, X-ray powder diffraction data provide more accurate $d$ values needed for precise unit-cell parameters. The complementarity of X-ray powder and electron diffraction methods is also seen in that the X-ray powder pattern is representative for the whole sample, whereas electron diffraction is collected from one crystal at a time. For multiphase samples, it may be impossible to index the X-ray powder diffraction pattern. Using only electron diffraction, on the other hand, may result in collecting data from a minority phase, mistaken for being the main phase (for example if the minority phase has thinner crystals that diffract electrons better). In such cases, it is invaluable to combine the two methods, making sure that the strongest diffraction spots in ED correspond to the strongest peaks in X-ray powder diffraction.

The simultaneous presence of diffraction data from the ZOLZ and first-order Laue zone (FOLZ) also helps in determining the space group by comparing the systematic absences in both the ZOLZ and FOLZ, especially when precession electron diffraction is applied. With electron precession, the FOLZ ring expands considerably, making it easy to see the lattice of diffraction spots. By comparing the lattices of spots in the ZOLZ and the FOLZ, it is straightforward to see if the lattice is primitive or centered, and to see if there is a glide plane perpendicular to the present direction. For primitive cells without glide planes, the ZOLZ and FOLZ lattices are identical. If there is a glide plane, half the reflections in the ZOLZ are absent. If the crystal lattice is centered, there are equally many spots per unit area in the ZOLZ and in the FOLZ, but the lattices are shifted relative to each other. All the details of how this can be used for (partial) spacegroup determination have been worked out by Morniroli \& Steeds (1992) and demonstrated on real crystals by Morniroli \& Redjaimia (2007). An example of determining the symmetry of the mineral mayenite is shown in Fig. 6. Convergent-beam electron diffraction can also be used for determination of unit cell and space group, see for example Williams \& Carter (1996).

\subsection{Phasing electron diffraction data}

The phase information which is lost in electron diffraction can be retrieved by direct methods, just as for X-rays. This is especially useful when it is not possible to obtain any (or sufficiently many) reflections with phases from HRTEM images to solve the structure. Some reasons may be: radiation damage of the sample, crystals too thick, insufficient resolution of the electron microscope. Direct methods have been applied to electron diffraction data, as first introduced by

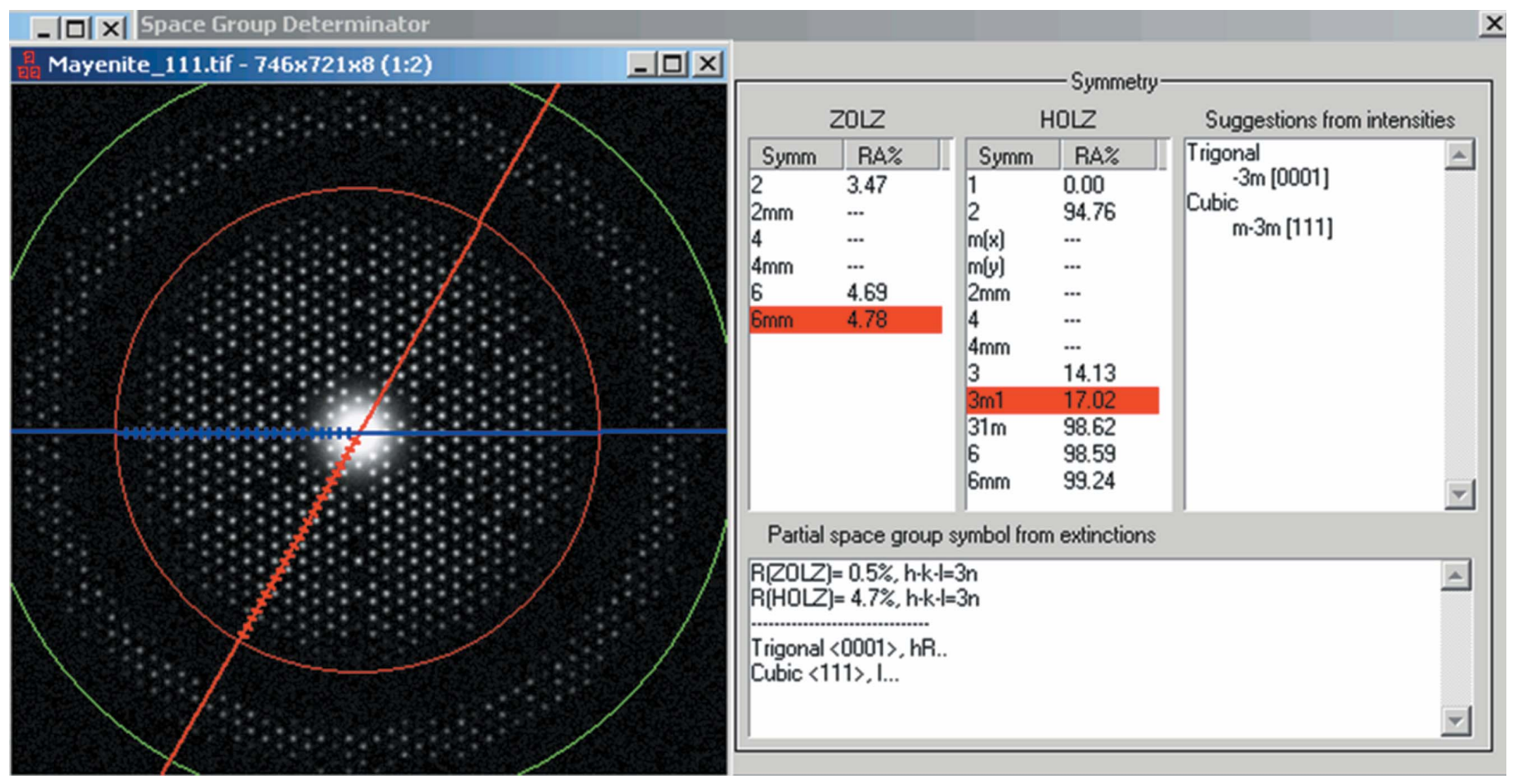

Figure 6

Precession diffraction pattern of mayenite (cubic, $I \overline{4} 3 d, a=11.99 \AA$ ) simulated by eMap (Oleynikov, 2006) and analyzed by Space Group Determinator (Oleynikov et al., 2008). The symmetry of the inner ZOLZ is $6 \mathrm{~mm}$ but the HOLZ ring is only $3 \mathrm{~m}$, ruling out hexagonal space groups. The extinction rules $(h+k-l=3 n)$ are only compatible with rhombohedral and cubic symmetries. Since the crystal is cubic, the zone axis must be [111] and the space group must be $I$-centered. 
Dorset \& Hauptman (1976) and recently reviewed by Gilmore (2003), who also developed the method using maximum entropy and likelihood phasing for solving structures from ED data (Gilmore et al., 1990). Electron diffraction data are less ideal for this purpose than X-ray data for two reasons; multiple scattering of electrons makes the relative intensities uncertain and the electron diffraction data sets are seldom complete. In spite of these problems, it has recently been shown that inorganic crystal structures with quite heavy elements can also be solved by direct methods provided that the crystals are thin enough (Wagner et al.,1999; Weirich et al., 2000).

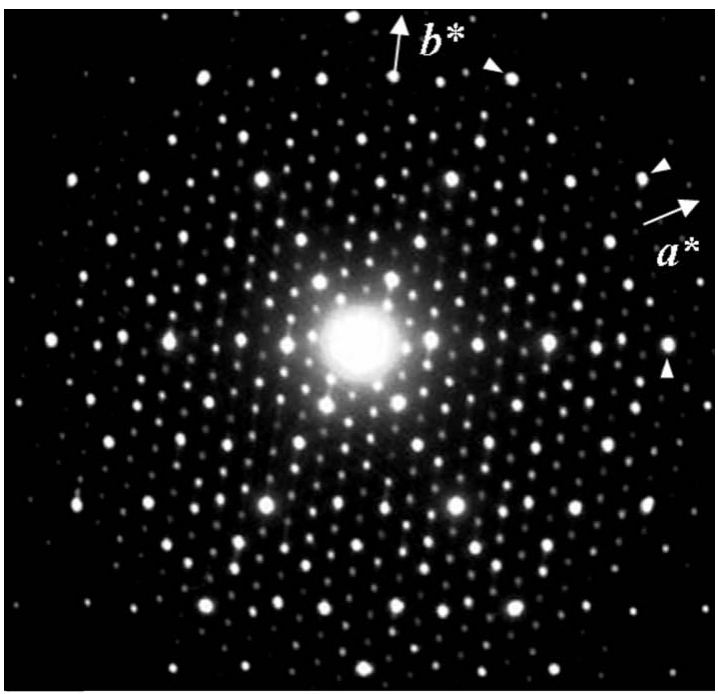

(a)

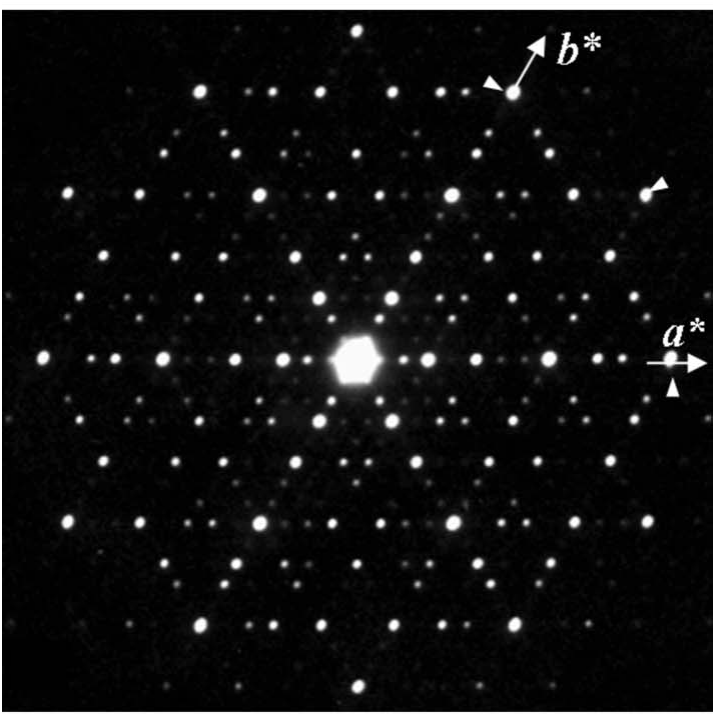

(b)

\section{Figure 7}

Electron diffraction patterns from $(a)$ the $\lambda$ and $(b)$ the $\tau(\mu)$ approximants taken at $100 \mathrm{kV}$ along the $c$ axis, on the same scale and with similar orientation. The corresponding strongest diffraction spots in the two approximants are marked by arrow heads. The diffraction pattern of $\lambda$ in $(a)$ shows only $p 6$ symmetry while that of $\tau(\mu)$ in $(b)$ shows $p 6 m$ symmetry. Note however that the strongest diffraction spots in $\lambda(a)$ have approximate $p 6 m$ symmetry. From Zhang, He et al. (2006).
The precession electron diffraction technique by Vincent \& Midgley (1994) can minimize the contribution from the nonsystematic multiple scattering and push the limit of direct phasing of electron diffraction data. Gjønnes and co-workers (Gjønnes, Cheng et al., 1998; Gjønnes, Hansen et al., 1998) solved the structure of $\mathrm{Al}_{m} \mathrm{Fe}$ from three-dimensional precession diffraction data using Patterson methods. Gemmi $e t$ al. (2003) solved the structure of $\mathrm{Ti}_{2} \mathrm{P}$ by direct methods using precession data merged from three projections. Own has built his own precession systems and made systematic studies on the applicability and limitations of precession data for structure determination (Own, 2005). Only after the more recent development of a commercially available electron precession camera, the Spinning Star by NanoMEGAS (2006), has precession electron diffraction data been applied to a wide range of inorganic structures, for example perovskite-related heavy-metal oxides (Boulahya et al., 2007), minerals (Gemmi \& Nicolopoulos, 2007) and zeolites (Dorset et al., 2007). Different methods such as direct methods, Patterson methods and maximum entropy and likelihood were used for phasing the diffraction data.

A new way for phasing ED data of a series of structurally related compounds, such as quasicrystal approximants, is to use the 'the strong reflections approach' (Christensen et al., 2004). This approach is valid on different structures that contain similar scattering motifs (for example atom clusters) oriented in a similar way, giving similar intensity distribution of reflections in reciprocal space. The structure-factor phases of the strong reflections of an unknown structure are deduced from those of a known related structure, while the structurefactor amplitudes of the unknown structure can be obtained either experimentally, by X-ray or electron diffraction, or estimated from those of the known related structure. Atomic positions are obtained directly from the three-dimensional map calculated from the obtained structure-factor amplitudes and phases of the strong reflections. The strong-reflections approach is based on three important facts: (i) for solving structures, only the strongest reflections are needed and their structure-factor phases must be correct; (ii) when the strongest reflections with correct phases are included in the calculation of the three-dimensional map, the structure model obtained is correct to the extent that $90-100 \%$ of the heaviest atoms are found within about $0.2 \AA$ from their correct positions; (iii) for quasicrystal approximants that are closely related, where the strong reflections are close to each other in reciprocal space and have similar structure-factor amplitudes (as in Fig. 7), their phases will also be similar (Zhang, Zou et al., 2006; Zhang, He et al., 2006). The strong-reflection approach has been used to solve structures of several unknown quasicrystal approximants (Oleynikov et al., 2006; Zhang, He et al., 2006; He et al., 2007).

\section{Structure refinement}

Complete crystal-structure determination (after collecting the data and finding the unit cell and symmetry) can be divided into two distinct steps: solving the structure and refining it. 
Solving the structure means finding a rough model of at least the strongest scattering (= heaviest) atoms. For solving a structure, any method, including trial and error, may be used. The structure model arrived at, from for example HRTEM images, needs to be verified and further improved. This can be done by refinement of the structure model by least-squares methods against ED data. The accuracy of atomic coordinates obtained from a two-dimensional projection by TEM to $2 \AA$ resolution is about $0.1-0.2 \AA$. This limited accuracy is mainly due to the sparse amount of experimental data, with typically no more than one reflection per atomic coordinate. Electron diffraction data extend to much higher resolution, typically 0.8 to $0.5 \AA$ and so contain about ten times more reflections than the corresponding HRTEM image. ED data have the further advantage compared to the image data that the ED amplitudes are not distorted by the contrast transfer function (CTF) of the microscope and do not change dramatically with the defocus as do the images. The fact that ED patterns do not contain phase information is no disadvantage for the refinement because only the amplitudes are needed at this stage of a structure determination.

Structure refinement based on kinematical scattering was first applied by Vainshtein (Vainshtein, 1956; Vainshtein et al., 1992). Weirich et al. (1996) solved the structure of the unknown compound $\mathrm{Ti}_{11} \mathrm{Se}_{4}$ from a HRTEM image combined with crystallographic image processing. 23 unique atoms were located from the reconstructed potential map. The structure was then refined from the crystallographic $R$ value of $48.4 \%$ of the starting model to $14.7 \%$ for the final model and a precision of $0.02 \AA$ for all the atoms using intensities extracted from ED patterns (extending to $0.75 \AA$ resolution) of a very thin crystal.

If the crystals are thick, structure refinement assuming the kinematical approximation is no longer possible. ED data contain a higher proportion of multiply scattered electrons than HRTEM images, mainly because ED patterns are usually taken from a larger and thicker region than those thinnest parts of HRTEM images which are cut out and used for image processing. As a remedy for this, structure refinement taking the dynamical scattering into account was developed by Sha $e t$ al. (1993) and Zandbergen and Jansen (Zandbergen et al., 1997; Jansen et al., 1998), the latter known as the MSLS (for multislice least squares) software. Electron diffraction from crystal regions with relatively homogeneous thicknesses was used. The crystal orientation, crystal thickness and the atomic coordinates were refined simultaneously. The advantage of MSLS is that the method can be applied to thicker crystals. However, the electron diffraction patterns should be taken from areas with homogeneous crystal thickness and free of crystal bending. Very often, a strong and coherent electron source provided by for example FEG is needed. The computation time also increases drastically with the complexity of structures.

Another approach for structure solution and refinement is to use the dynamical diffraction data from convergent-beam electron diffraction (Zuo \& Spence, 1991; Tsuda \& Tanaka, 1995). Accurate structure-factor determination by CBED can provide information on the location of valence-charge distri- bution (Zheng et al., 2005). Structure analysis by CBED has been summarized in two review articles (Spence, 1993; Tanaka, 1994).

Structure refinement by X-ray powder diffraction using the Rietveld method has become a routine method. When good enough X-ray powder diffraction data are available, it is very efficient to refine the structures solved by electron crystallography against X-ray powder diffraction data (Wagner et al., 1999).

\section{Ultrafast electron crystallography}

Most chemical reactions and phase transitions occur on a time scale of femto- to picoseconds. The strong interaction between electrons and matter makes electron crystallography the unique technique to determine the entire structure changes within such a short time scale. A new field of electron crystallography, four-dimensional ultrafast electron crystallography (UEC), has been developed by Zewail's group (Ihee et al., 2001; Zewail, 2006) and Siwick et al. (2003) to combine atomic scale resolutions in both space and time. The main advantages of using electrons to achieve joint spatial $(\AA)$ and temporal (ps and fs) resolutions compared to X-rays are: $(a)$ the cross section for electron scattering is about six orders of magnitude larger than that of X-ray scattering, so electrons can reveal transient structures of gases, surfaces and (thin) crystals; (b) electrons are less damaging to specimens per useful elastic scattering event than are X-rays (Henderson, 1995); (c) electrons can be focused to form images, $(d)$ the experiments are of 'tabletop' scale and can be implemented with ultrafast (fs and ps) laser sources, and (e) with properly timed sequences of electron pulses - frame referencing - it is possible to 'isolate' in time the evolving transient structure(s) (Zewail, 2006). This makes electrons more feasible for structural studies on a femto- and picosecond scale compared to the 'free-electron' synchrotron source (Thomas, 2004).

Ultrafast electron crystallography has been applied to transient structures of molecules, surfaces and phase transitions (Ruan, Vigliotti et al., 2004), structural dynamics of interfacial water on hydrophilic and hydrophobic substrates (Ruan, Lobastov et al., 2004), monolayer and bilayer phospholipids on hydrophilic and hydrophobic substrates (Chen et al., 2006), surface structural dynamics with atomic scale resolution (Vigliotti et al., 2004; Chen et al., 2005), and structures and dynamics of self-assembled surface monolayers (Ruan, Yang \& Zewail, 2004). Applications of this technique will open a new era in physics, chemistry and biology.

\section{Conclusions}

Electron crystallography has proven to be a unique technique for atomic structure determination of nano-sized crystals, from inorganic through organic to protein crystals. There is no overlapping problem of diffraction peaks from nano-sized single crystals as in X-ray powder diffraction. Crystal structures can be directly determined from HRTEM images of thin crystals, from which both the structure-factor amplitude and 
phase information can be obtained by crystallographic image processing. Crystal structures can be solved to atomic resolution in two dimensions as well as in three dimensions, and in principle there is no limitation to the complexity of the structures. Structures can also be determined by exit-wave reconstruction from a through-focus series of HRTEM images. In addition, HAADF STEM imaging gives $Z$-contrast that can also be used for the location of heavy atoms. While crystallographic image processing mainly deals with crystalline samples, exit-wave reconstruction and HAADF STEM imaging can be used for studying also non-crystalline samples, defects and interfaces. Since all this information is related to the atomic structures of the sample, crystallographic image processing can be applied to the reconstructed exit waves and HAADF STEM images of crystals.

Atomic structures can be determined from only electron diffraction data. The phase information can then be obtained by for example direct methods, Patterson methods, maximum entropy and likelihood, and from related structures by the strong reflections approach. The development of the electron precession technique makes structure determination by electron diffraction very promising. Recent developments in instrumentation and methods have not only pushed the spatial resolution of electron crystallography into the sub-ångström scale but also the time scale into femtoseconds.

However, electron crystallography is still a complementary method to X-ray diffraction. Before electron crystallography can become a routine technique for crystal-structure determination, as single-crystal X-ray diffraction and X-ray powder diffraction are today, several major challenges remain. Automatic collection of high-quality three-dimensional data, automatic data processing and methods, and software development for routine structure determination must be developed. Significant advances have been made towards these goals during the past decade. Hopefully, routine structure determination by electron crystallography is just around the corner.

We dedicate this paper to the memory of Professor Kehsin Kuo who made important contributions in electron crystallography. We thank all collaborators in the past and present for fruitful collaboration, and the Wenner-Gren Foundation and Carl-Tyggers Foundation for several post doc grants. This work was supported by the Swedish Research Council (VR). The Berzelii Centre EXSELENT is financially supported by VR and the Swedish Governmental Agency for Innovation Systems (VINNOVA).

\section{References}

Baerlocher, C., Gramm, F., Massüger, L., McCusker, L. B., He, Z. B., Hovmöller, S. \& Zou, X. D. (2007). Science, 315, 1113-1116.

Boulahya, K., Ruiz-González, L., Parras, M., González-Calbet, J. M., Nickolsky, M. S. \& Nicolopoulos, S. (2007). Ultramicroscopy, 107, 445-452.

Chen, S., Seidel, M. T. \& Zewail, A. H. (2005). Proc. Natl Acad. Sci. USA, 102, 8854-8859.
Chen, S., Seidel, M. T. \& Zewail, A. H. (2006). Angew. Chem. Int. Ed. 45, 5154-5158.

Christensen, J., Oleynikov, P., Hovmöller, S. \& Zou, X. D. (2004). Ferroelectrics, 305, 273-277.

Coene, W. M. J., Janssen, G., Op de Beeck, M. \& Van Dyck, D. (1992). Phys. Rev. Lett. 69, 3743-3746.

Coene, W. M. J., Thust, A., Op de Beeck, M. \& Van Dyck, D. (1996). Ultramicroscopy, 64, 109-135.

Cowley, J. M. (1953a). Acta Cryst. 6, 516-521.

Cowley, J. M. (1953b). Acta Cryst. 6, 522-529.

Cowley, J. M. (1953c). Acta Cryst. 6, 846-853.

Cowley, J. M. \& Moodie, A. F. (1957). Acta Cryst. 10, 609-619.

Cowley, J. M. \& Moodie, A. F. (1959a). Acta Cryst. 12, 353-359.

Cowley, J. M. \& Moodie, A. F. (1959b). Acta Cryst. 12, 360-367.

Crowther, R. A., Amos, L. A., Finch, J. T., DeRosier, D. J. \& Klug, A. (1970). Nature (London), 226, 421-425.

DeRosier, D. J. \& Klug, A. (1968). Nature (London), 217, 130-134.

Dorset, D. L. (1995). Structural Electron Crystallography. New York: Plenum.

Dorset, D. L. (1998). Acta Cryst. A54, 750-757.

Dorset, D. L. (2007). Ultramicroscopy, 107, 453-461.

Dorset, D. L., Gilmore, C. J., Jorda, J. L. \& Nicolopoulos, S. (2007). Ultramicroscopy, 107, 462-473.

Dorset, D. L. \& Hauptman, H. A. (1976). Ultramicroscopy, 1, 195-201.

Gao, C. B., Sakamoto, Y., Sakamoto, K., Terasaki, O. \& Che, S. (2006). Angew. Chem. Int. Ed. 45, 4295-4298.

Garcia-Bennett, A. E., Miyasaka, K., Terasaki, O. \& Che, S. (2004). Chem. Mater. 16, 3597-3605.

Garcia-Bennett, A. E., Terasaki, O., Che, S. \& Tatsumi, T. (2004). Chem. Mater. 16, 813-821.

Gemmi, M. \& Nicolopoulos, S. (2007). Ultramicroscopy, 107, 483-494.

Gemmi, M., Zou, X. D., Hovmöller, S., Migliori, A., Vennström, M. \& Andersson, Y. (2003). Acta Cryst. A59, 117-126.

Gilmore, C. J. (2003). Crystallogr. Rev. 9, 17-31.

Gilmore, C. J., Bricogne, G. \& Bannister, C. (1990). Acta Cryst. A46, 297-308.

Gjønnes, J., Hansen, V., Berg, B. S., Runde, P., Cheng, Y. F., Gjønnes, K., Dorset, D. L. \& Gilmore, J. C. (1998). Acta Cryst. A54, 306-319.

Gjønnes, K., Cheng, Y., Berg, B. S. \& Hansen, V. (1998). Acta Cryst. A54, 102-119.

Gramm, F., Baerlocher, Ch., McCusker, L. B., Warrender, S. J., Wright, P. A., Han, B., Hong, S. B., Liu, Z., Ohsuna, T. \& Terasaki, O. (2006). Nature (London), 444, 79-81.

Grosse-Kunstleve, R. W., McCusker, L. B. \& Baerlocher, Ch. (1997). J. Appl. Cryst. 30, 985-995.

Grosse-Kunstleve, R. W., McCusker, L. B. \& Baerlocher, C. (1999). J. Appl. Cryst. 32, 536-542.

Haider, M., Rose, H., Uhlemann, S., Schwan, E. \& Urban, K. (1998). Nature (London), 392, 768-769.

He, Z. B., Zou, X. D., Hovmöller, S., Oleynikov, P. \& Kuo, K. H. (2007). Ultramicroscopy, 107, 495-500.

Henderson, R. (1995). Q. Rev. Biophys. 28, 171-193.

Henderson, R. \& Unwin, P. N. T. (1975). Nature (London), 257, 28-32.

Hovmöller, S. (1992). Ultramicroscopy, 41, 121-135.

Hovmöller, S., Sjögren, A., Farrants, G., Sundberg, M. \& Marinder, B.-O. (1984). Nature (London), 311, 238-241.

Hovmöller, S. \& Zou, X. D. (1999). Microsc. Res. Tech. 46, 147-159.

Hu, J. J., Li, F. H. \& Fan, H. F. (1992). Ultramicroscopy, 41, 387-397.

Ihee, H., Lobastov, V. A., Gomez, U. M., Goodson, B. M., Srinivasan, R., Ruan, C. Y. \& Zewail, A. H. (2001). Science, 291, 458-462.

Iijima, S. (1971). J. Appl. Phys. 42, 5891-5893.

Jansen, J., Tang, D., Zandbergen, H. W. \& Schenk, H. (1998). Acta Cryst. A54, 91-101.

Jia, C. L., Lentzen, M. \& Urban, K. (2003). Science, 299, 870-873.

Jia, C. L. \& Urban, K. (2004). Science, 303, 2001-2004. 
Kaneda, M., Tsubakiyarna, T., Carlsson, A., Sakamoto, Y., Ohsuna, T., Terasaki, O., Joo, S. H. \& Ryoo, R. (2002). J. Phys. Chem. B, 106, $1256-1266$.

Klug, A. (1978-79). Chim. Scr. 14, 245 -256.

Knoll, M. \& Ruska, E. (1932). Z. Phys. 78, 318-339.

Köhler, J., Svensson, G. \& Simon, A. (1992). Angew. Chem. Int. Ed. 31, 1437-1456.

Kolb, U., Gorelik, T., Kübel, C, Otten, M. T. \& Hubert, D. (2007). Ultramicroscopy, 107, 507-513.

Leonowicz, M. E., Lawton, J. A., Lawton, S. L. \& Rubin, M. K. (1994). Science, 264, 1910-1913.

Leslie, C., Landree, E., Collazo-Davila, C., Bengu, E., Grozea, D. \& Marks, L. D. (1999). Microsc. Res. Tech. 46, 160-177.

Lichte, H., Formanek, P., Lenk, A., Linck, M., Matzeck, Ch., Lehmann, M. \& Simon, P. (2007). Annu. Rev. Mater. Res. 37, 539-588.

Liu, Z., Ohsuna, T., Terasaki, O., Camblor, M. A., Diaz-Cabanas, M. J. \& Hiraga, K. (2001). J. Am. Chem. Soc. 123, 5370-5371.

Mo, Y. D., Cheng, T. Z., Fan, H. F., Li, J. Q., Sha, B. D., Zheng, C. D., Li, F. H. \& Zhao, Z. X. (1992). Supercond. Sci. Technol. 5, $69-72$.

Morniroli, J.-P. \& Redjaimia, A. J. (2007). J. Microsc. 227, 157-171.

Morniroli, J.-P. \& Steeds, J. (1992). Ultramicroscopy, 45, 219-239.

NanoMEGAS (2006). http://www.nanomegas.com/.

Nellist, P. D., Chisholm, M. F., Dellby, N., Krivanek, O. L., Murfitt, M. F., Szilagyl, Z. S., Lupini, A. R., Borisevich, A., Sides, W. H. \& Pennycook, S. J. (2004). Science, 305, 1741.

Oleynikov, P. (2006). http://www.analitex.com/Index.html.

Oleynikov, P., Demchenko, L., Christensen, J., Hovmöller, S., Yokosawa, T., Döblinger, M., Gruschko, B. \& Zou, X. D. (2006). Philos. Mag. 86, 457-462.

Oleynikov, P., Zou, X. D. \& Hovmöller, S. (2008). In preparation.

Own, C. S. (2005). System Design and Verification of the Precession Electron Diffraction Technique. PhD thesis, Northwestern University, Chicago, USA.

Palatinus, L. \& Chapuis, G. (2006). http://superspace.epfl.ch/superflip. Pennycook, S. J. \& Jesson, D. E. (1991). Ultramicroscopy, 37, 14-38.

Pinsker, Z. G. (1949). Diffraktsiya Elektronov. Moscow/Leningrad: Akad. Nauk SSSR Press. Engl. transl: Electron Diffraction (1953). London: Butterworths.

Porter, K. R., Claude, A. \& Fullam, E. F. (1945). J. Exp. Med. 81, 233-246.

Renault, L., Chou, H. T., Chiu, P.-L., Hill, R. M., Zeng, X. G., Gipson, B., Zhang, Z. Y., Cheng, A. C., Unger, V. \& Stahlberg, H. (2006). J. Comput. Aided Mol. Des. 20, 519-527.

Ruan, C. Y., Lobastov, V. A., Vigliotti, F., Chen, S. \& Zewail, A. H. (2004). Science, 304, 80-84.

Ruan, C.-Y., Vigliotti, F., Lobastov, V. A., Chen, S. \& Zewail, A. H. (2004). Proc. Natl Acad. Sci. USA, 101, 1123-1128.

Ruan, C.-Y., Yang, D.-S. \& Zewail, A. H. (2004). J. Am. Chem. Soc. 126, 12797-12799.

Sakamoto, Y., Diaz, I., Terasaki, O., Zhao, D. Y., Perez-Pariente, J., Kim, J. M. \& Stucky, G. D. (2002). J. Phys. Chem. B, 106, 3118-3123.

Sakamoto, Y., Kaneda, M., Terasaki, O., Zhao, D. Y., Kim, J. M., Stucky, G. D., Shin, H. J. \& Ryoo, R. (2000). Nature (London), 408, 449-453.

Sakamoto, Y., Kim, T. W., Ryoo, R. \& Terasaki, O. (2004). Angew. Chem. Int. Ed. 43, 5231-5234.

Sha, B. D., Fan, H. F. \& Li, F. H. (1993). Acta Cryst. A49, 877-880.

Siwick, B. J., Dwyer, J. R., Jordan, R. E. \& Dwayne Miller, R. J. (2003). Science, 302, 1382-1385.

Spence, J. C. H. (1993). Acta Cryst. A49, 231-260.

Sun, J.-L., He, Z. B., Hovmöller, S., Zou, X. D., Gramm, F., Baerlocher, Ch. \& McCusker, L. B. (2007). In preparation.

Svensson, G. (1990). Microsc. Microanal. Microstruct. 1, 343-356.
Tanaka, M. (1994). Acta Cryst. A50, 261-286.

Thomas, J. M. (2004). Angew. Chem. Int. Ed. 43, 2606-2610.

Tonomura, A. (1999). Electron Holography. Optical Sciences Series. Berlin: Springer.

Tsuda, K. \& Tanaka, M. (1995). Acta Cryst. A51, 7-19.

Unwin, P. N. T. \& Henderson, R. (1975). J. Mol. Biol. 94, 425-440.

Vainshtein, B. K. (1956). Strukturnaya Elektronographiya. Moscow: Izd'vo Akad. Nauk SSSR. Engl. transl: Structure Analysis by Electron Diffraction (1964). Oxford: Pergamon Press.

Vainshtein, B. K., Zvyagin, B. B. \& Avilov, A. S. (1992). Electron Diffraction Techniques, Vol. 1, edited by J. M. Cowley, p. 216. Oxford University Press.

Van Dyck, D. \& Op de Beeck, M. (1990). Proceedings of XII International Congress on Electron Microscopy, Seattle, USA, pp. 26-27.

Vigliotti, F., Chen, S., Ruan, C.-Y., Lobastov, V. A. \& Zewail, A. H. (2004). Angew. Chem. Int. Ed. 43, 2705-2709.

Vincent, R. \& Midgley, P. A. (1994). Ultramicroscopy, 53, 271-282.

Völkl, E., Allard, L. F. \& Joy, D. C. (1999). Editors. Introduction to Electron Holography. New York: Plenum Press.

Wagner, P., Terasaki, O., Ritsch, S., Nery, J. G., Zones, S. I., Davis, M. E. \& Hiraga, K. (1999). J. Phys. Chem B, 103, 8245-8250.

Wang, D. N., Hovmöller, S., Kihlborg, L. \& Sundberg, M. (1988). Ultramicroscopy, 25, 303-316.

Weirich, T. E., Ramlau, R., Simon, A., Hovmöller, S. \& Zou, X. D. (1996). Nature (London), 382, 144-146.

Weirich, T. E., Zou, X. D., Ramlau, R., Simon, A., Cascarano, G. L., Giacovazzo, C. \& Hovmöller, S. (2000). Acta Cryst. A56, 29-35.

Wenk, H.-R., Downing, K. H., Hu, M. \& O'Keefe, M. A. (1992). Acta Cryst. A48, 700-716.

Williams, D. B. \& Carter, C. B. (1996). Transmission Electron Microscopy: Diffraction, Vol. 2. New York: Plenum Press.

Wu, L. J., Zhu, J. M., Vogt, T., Su, H. B., Davenport, J. W. \& Tafto, J. (2004). Phys. Rev. B, 69, 064501.

Xiang, S.-B., Li, F.-H. \& Fan, H.-F. (1990). Acta Cryst. A46, 473-478.

Yu, T., Zhang, H., Yan, X. W., Chen, Z. X., Zou, X. D., Oleynikov, P. \& Zhao, D. Y. (2006). J. Phys. Chem. B, 110, 21467-21472.

Zandbergen, H. W., Andersen, S. J. \& Jansen, J. (1997). Science, 277, 1221-1224.

Zewail, A. H. (2006). Annu. Rev. Phys. Chem. 57, 65-103.

Zhang, H., He, Z. B., Oleynikov, P., Zou, X. D., Hovmöller, S. \& Kuo K. H. (2006). Acta Cryst. B62, 16-25.

Zhang, H., Zou, X. D., Oleynikov, P. \& Hovmöller, S. (2006). Philos. Mag. 86, 543-548.

Zheng, J.-C., Zhu, Y., Wu, L. \& Davenport, J. W. (2005). J. Appl. Cryst. 38, 648-656.

Zou, X. D. (1995). Electron Crystallography of Inorganic Structures. $\mathrm{PhD}$ thesis, Stockholm University, Sweden.

Zou, X. D. (1999). Microsc. Res. Tech. 46, 202-219.

Zou, X. D., Ferrow, E. A. \& Hovmöller, S. (1995). Phys. Chem. Miner. 22, 517-523.

Zou, X. D., Hovmöller, A. \& Hovmöller, S. (2004). Ultramicroscopy, 98, 187-193.

Zou, X. D., Mo, Z. M., Hovmöller, S., Li, X. Z. \& Kuo, K. H. (2003). Acta Cryst. A59, 526-539.

Zou, X. D., Sundberg, M., Larine, M. \& Hovmöller, S. (1996). Ultramicroscopy, 62, 103-121.

Zubkov, V. G., Pereleyaev, V. A., Tyutyunnik, A. P., Kontsevaya, I. A., Voronin, V. I. \& Svensson, G. (1994). J. Alloys Compd. 203, 209-216.

Zuo, J. M., Kim, M., O'Keffee, M. \& Spence, J. C. H. (1999). Nature (London), 401, 49-52.

Zuo, J. M. \& Spence, J. C. H. (1991). Ultramicroscopy, 35, 185-196.

Zvyagin, B. B. (1967). Electron Diffraction Analysis of Clay Mineral Structures. New York: Plenum Press. 